\title{
Energy Performance Evaluation of Hybrid VRF Systems Based on Japanese Government-Designated Method
}

\author{
Tokimi Kawase ${ }^{1}$, Atsushi Hashimoto ${ }^{1}$, Ken Yasuda $^{2}$, and Tatsuo Nobe ${ }^{1}$ \\ ${ }^{1}$ Kogakuin University, Tokyo, Japan \\ ${ }^{2}$ TOKYO GAS Company, Tokyo, Japan
}

\begin{abstract}
Variable Refrigerant Flow Rate (VRF) System is used as air-conditioning systems in small and medium-sized buildings in Japan. Traditionally, the two most common types of VRF systems are electric motor driven VRF Systems (EHP) and gas engine driven VRF Systems (GHP). However, in recent years' society is increasingly demanding energy saving and the best energy mix. This requires an air-conditioning system that has energy flexibly depending on the application and situation. As a result, a hybrid airconditioning system (called Hybrid VRF System) that satisfies theses need has been developed. The developed system can operate according to energy price and usage situations by connecting GHP and EHP with one refrigerant cycle. In recent years, a Hybrid VRF Systems have been used for small to mediumsized buildings. In this paper, the author evaluated the performance of an integrated Hybrid VRF System based on the energy evaluation method prescribed by Japanese law.
\end{abstract}

\section{Introduction}

Aiming to realize stable energy supply that is less burdensome on society and that operates as an energy supply and demand structure is an essential condition for the development of Japan. Therefore, Zero Energy Buildings (ZEBs) are gaining attention because they can realize substantial energy savings without deteriorating the environmental quality inside and outside the building. Since the Great East Japan Earthquake that occurred in 2011, demand for energy saving and the best energy mix is increasing in Japanese society as a whole against the backdrop of the decline in the power generation capacity of the electric power system. In recent years, the liberalization of electricity and gas markets has been implemented. As a result, it seems that various contracts will appear, and the importance priced for air-conditioning systems that can freely select energy according to circumstances has increased. Based on this background, an integrated Hybrid VRF System having an electric motor driven VRF System (EHP) and a gas engine driven VRF System (GHP) in the same refrigerant system was developed. Also an evaluation method index, an energy consumption performance calculation program, non-residential version (Web Program), was used. The Web Program is a calculation support program that can be used on a Web browser. It was used as an energy evaluation method based on Japanese laws and ordinances. In this paper, the author introduces an integrated Hybrid VRF System to a virtual medium sized office. The author evaluated the performance of the integrated Hybrid VRF System using the energy evaluation method Web Program established by Japanese law.

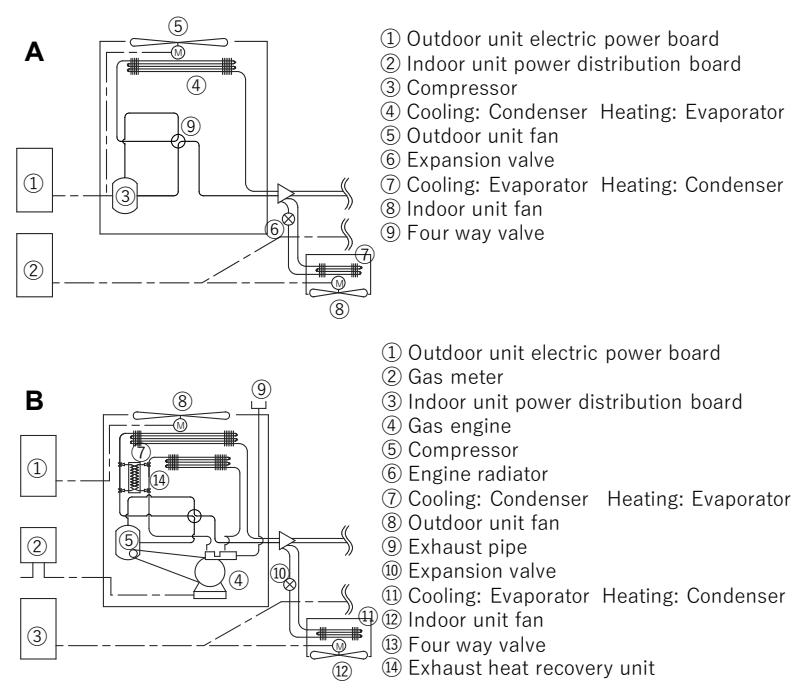

Fig. 1. Outdoor unit system diagram: (A) EHP (B)GHP

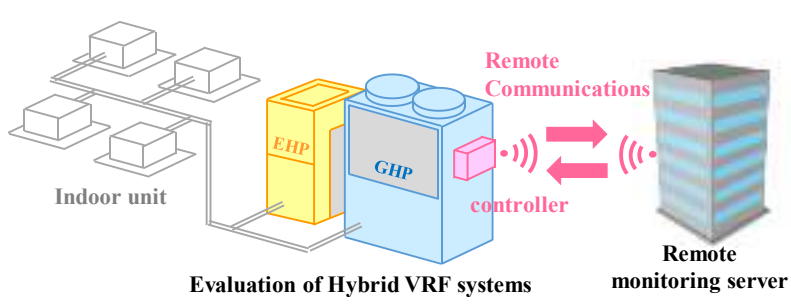

Fig. 2. Outline of the Hybrid VRF System

\footnotetext{
Tokimi Kawase: dm18017@ns.kogakuin.ac.jp
} 


\section{Hybrid VRF System configuration [1]}

Figure 1 shows the outdoor unit system diagram of the EHP and GHP. Figure 2 presents an outline of the integrated Hybrid VRF System. The Integrated Hybrid VRF System, GHP and EHP were connected to the same refrigerant system, and the operating ratio of GHP and EHP could be arbitrarily changed with respect to the air conditioning load demanded by the indoor unit. For changing the operation ratio, the author assumed that a controller with a remote communication function is installed in the customer property and is to be carried out by remote communication [2][3] from a remote monitoring server away from the customer property. In addition, by incorporating the electricity amount and the gas consumption amount of the entire property into the controller, it was possible to grasp the energy use situation of the entire property. The remote monitoring server monitors the energy demand and supply situation of the energy supply company and the momentary change of the energy price and calculates the optimum operation method of EHP and GHP in the present situation and instructs the controller. By adopting such a configuration, it is possible for optimum energy management on the customer side according to the changing energy demand and supply situation and the energy price.

\section{Government-Designated Method}

In Japan, the Energy Basic Plan is a plan to show the long-term direction of energy policies. The plan is reviewed every three years. Based on the Basic Energy Policy Law, established in 2002, it is formulated in accordance with basic policies such as "safety," "stable supply," "improvement of economic efficiency," and "adaptation to the environment." In April 2014, the author aiming to realize and promote ZEB by using and disseminating renewable energy such as wind power and photovoltaic power generation. In order to achieve policy goals, the ZEB Roadmap Committee was established in 2015 and ZEB Design Guidelines for Design Professionals (medium / small scale office) was released in 2017. The guidelines are based on the Law Concerning the Improvement of Energy Consumption Performance of Buildings (Building Energy Conservation Law) and the Law Concerning the Rational Use of Energy, et cetera, which established the National Institute of Research and Innovation, the Building Research Institute, the Web Program: all of based on technology concerning the evaluation of energy consumption performance (including crust) conforming to the announcement of the Energy Conservation Law (Energy Conservation Act). In this research, simulation was carried out using Web Program.

\subsection{Net-ZERO Energy Building [4]}

Figure 3 shows the ZEB Chart and Figure 4 shows the BEI calculation. In Japan, "by utilizing natural energy by adopting energy load and adopting passive technology, introducing highly efficient facility system, the author aims to achieve significant energy saving without lowering the quality of the indoor environment, and by introducing renewable energy, the building can be defined as a structure aimed at zero balance of the primary energy consumption of the year. ZEB that achieves energy saving of more than $100 \%$ net, "Nearly ZEB" that achieves a net of $75 \%$ or more, and "ZEB Ready" that achieves a net of $50 \%$ or more are stipulated. It is based on the Building Energy Index(BEI) which is the ratio of the primary energy consumption of the design building compared with the base building based on the Web Program. When BEI $\leqq 0.5$, it is determined that ZEB (including ZEB Ready) has been achieved. However, the target of this primary energy consumption is regarded as air conditioning equipment, ventilation equipment other than air conditioning equipment, lighting equipment, hot water supply equipment and elevator.

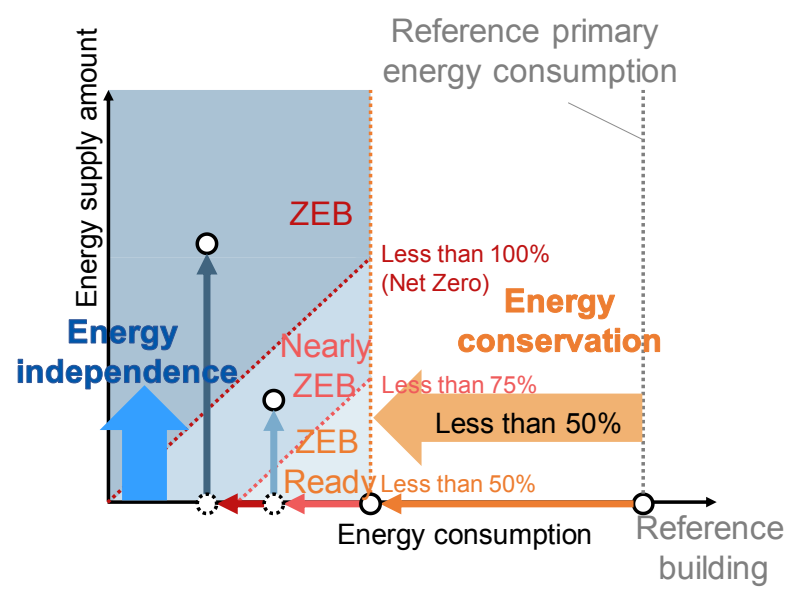

Fig. 3. ZEB chart

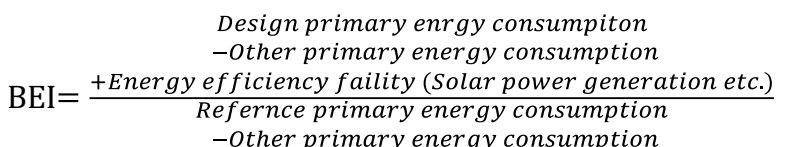

Fig. 4. BEI calculation

\subsection{Web Program}

Figure 5 shows the Web Program screen and Figure 6 shows the Input sheet constitution. The National Research and Development Corporation of Japan was established by the Building Research Institute on the basis of the FY 2016 standard. It is in compliance with the notice of Law Concerning Improvement of Energy Consumption Performance of Buildings (Building Energy Conservation Act) and Law Concerning Rationalization of Energy Use (Energy Conservation Act) is a calculation tool based on technology concerning the evaluation of energy consumption performance, including the hull. Web Program has exclusive Excel sheet, rough structure is classified into seven categories: common condition input, air conditioning equipment input, ventilation equipment input, lighting equipment 
input, hot water supply equipment input, elevator equipment and energy efficiency equipment. It is a specification automatically calculated by dragging and dropping an Excel sheet describing these conditions onto the Web Program. The results screen shows not only BEI but also PAL*(Perimeter Annual Load*) and energy consumption performance.

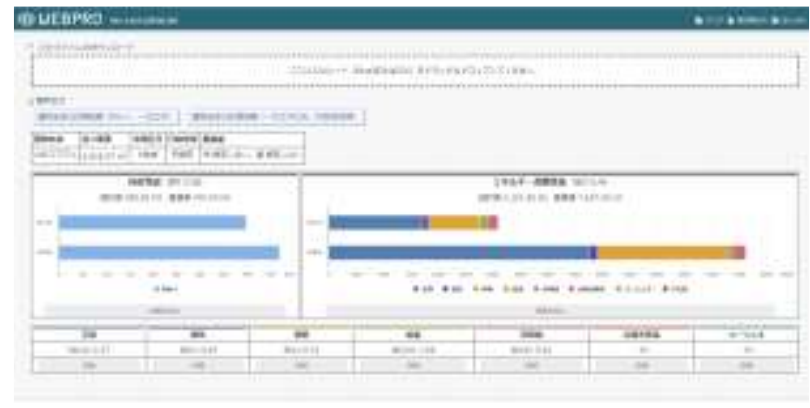

Fig. 5. Web Program screen [5]

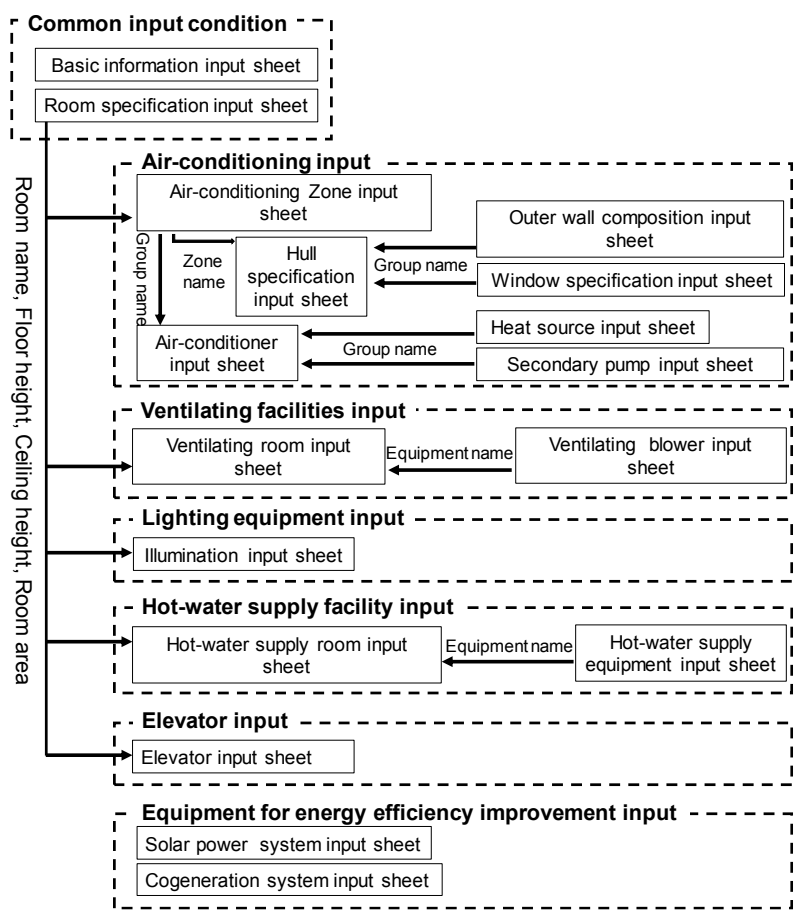

Fig. 6. Input sheet constitution

\subsubsection{Basis for Calculating Reference Primary Energy Consumption [6]}

Figure 7 shows the calculation target building model and Table 1 shows the calculation cases. These define the "standard setting outer skin specification" and "standard set air conditioning equipment specification" for room use and area. The author conducted an actual analysis of the energy conservation plan based on the Building Energy Conservcation Act, various literature, surveys and interviews with dosigners,etc. Based on the results, standard outer skin specifications and equipment specifications were defined as of 2013. The calculation of primary energy consumption covers the "building model. It depends greatly on the shape and orientation of the building and the dimentions of the outerskin surface. A total of 24 cases were taken into consideration and analyzed.

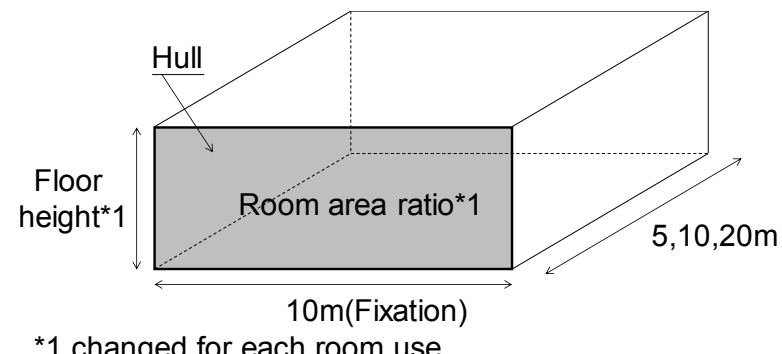

*1 changed for each room use

Fig. 7. Calculation target building model

Table 1. Calculation case

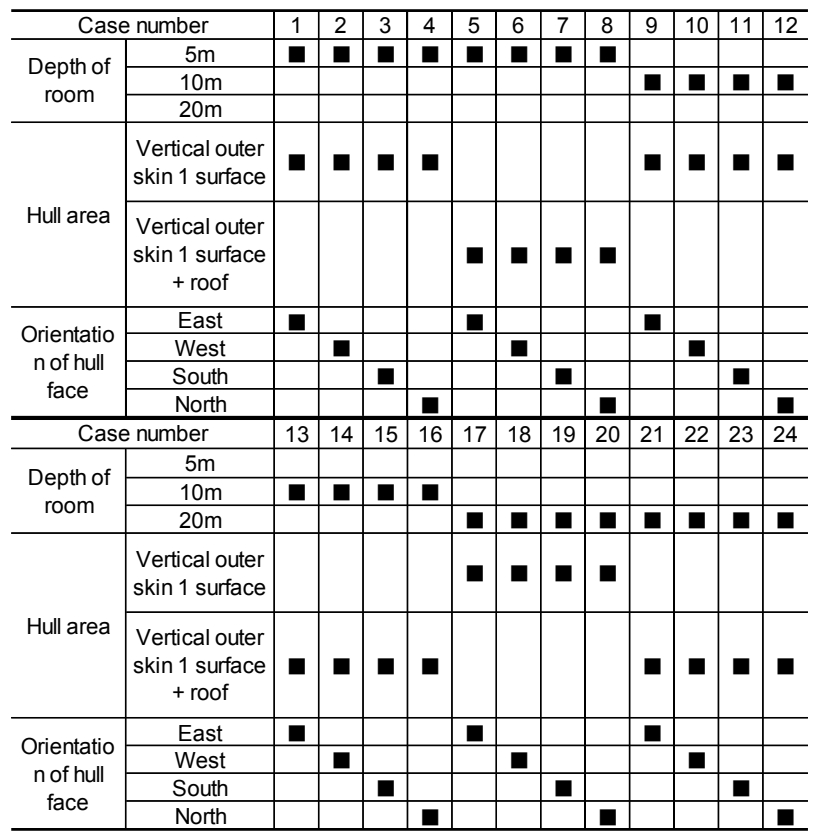

\section{Simulation Outline}

Table 2 shows the verification pattern, Table 3 shows the building specifications, Table 4 shows the input conditions, and Figure 8 shows the simulation flow chart. The virtual middle-sized office 2), described in the ZEB guidelines as a medium size office edition was targeted, and the verification pattern for air-conditioning capacities were 100, 150, and $200(\mathrm{~W} / \mathrm{m} 2$ ) determined by the cooling capacity. In order to make it suitable for these buildings, the north and south sides of the virtual medium-sized office, described in the ZEB design guidelines, were cut by $4(\mathrm{~m})$ to make a virtual mediumsized office with five stories above the ground. The airconditioning system was changed from the central type to the individual dispersion type, and other ventilation, lighting, hot water supply, and elevator equipment was simulated in the same way as the specifications of the ZEB design guidelines. 
Table 2. shows the verification pattern

\begin{tabular}{c|c|c|c}
\hline & $100\left[\mathrm{~W} / \mathrm{m}^{2}\right]$ & $150\left[\mathrm{~W} / \mathrm{m}^{2}\right]$ & $200\left[\mathrm{~W} / \mathrm{m}^{2}\right]$ \\
\hline i & not EHP number control & not EHP number control & not EHP number control \\
\hline ii & EHP number control & EHP number control & EHP number control \\
\hline iii & GHP number control & GHP number control & GHP number control \\
\hline iv & Hybrid VRF Systems & Hybrid VRF Systems & Hybrid VRF Systems \\
\hline
\end{tabular}

Table 3. shows the building specifications

\begin{tabular}{|c|c|}
\hline & Simulation contens \\
\hline $\operatorname{Rank[F]}$ & 5 \\
\hline Site area[m²] & 4570 \\
\hline Building area $\left[\mathrm{m}^{2}\right]$ & 1155 \\
\hline Total floor area[m²] & 5616.57 \\
\hline Air conditioning area[m²] & 4094.44 \\
\hline Location & OKAYAMA in Japan \\
\hline Weather date & $\begin{array}{c}\text { 1981～1995年 } \\
\text { Automated Meteorological Data } \\
\text { Acquisition System }\end{array}$ \\
\hline
\end{tabular}

Table 4. shows the input condition

\begin{tabular}{|c|c|}
\hline Hull & $\begin{array}{l}\text { Extrusion method } 3 \text { polystyrene } \\
\text { heat insulating plates } \\
(\text { roof: } 100(\mathrm{~mm}), \text { out wall: } 50(\mathrm{~mm}))\end{array}$ \\
\hline \multirow[t]{2}{*}{ Air conditioning } & $\begin{array}{l}\text { Individual distribution method } \\
\text { (package air conditioner / gas heat } \\
\text { pump air conditioner) }\end{array}$ \\
\hline & $\begin{array}{l}\begin{array}{l}\text { Indoor unit } \\
\text { (cooling capacity } 5.6(\mathrm{~kW}) / \text { heating }\end{array} \\
\end{array}$ \\
\hline Ventilation equipment & $\begin{array}{l}\text { High effciency motor / temperature } \\
\text { control / inverter } \\
\text { introduction) }\end{array}$ \\
\hline Lighting equipment & $\begin{array}{l}1252 \text { unitis } \\
\text { LED lighting } \\
\text { ( brightness detection control / time } \\
\text { schedule control / initial } \\
\text { illumination correction control) }\end{array}$ \\
\hline $\begin{array}{c}\text { Hot water } \\
\text { supply equipment }\end{array}$ & $\begin{array}{l}\text { Local electric storage type } \\
\text { (rated heating capacity } 1.1(\mathrm{~kW}) / \\
\text { heat source efficiency } 0.37)\end{array}$ \\
\hline Elevator & $\begin{array}{l}2 \text { units } \\
\text { Load capacity } 800(\mathrm{~kg}) \\
\text { speed } 60(\mathrm{~m} / \mathrm{min}) \\
\text { VVF } \\
\text { (with electric regeneration, } \\
\text { gearless) }\end{array}$ \\
\hline
\end{tabular}

\begin{tabular}{|c|c|}
\hline \multicolumn{2}{|c|}{ Virtual medium sized office } \\
\hline 7 & 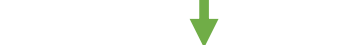 \\
\hline $\begin{array}{l}\text { Setting outdoor unit } \\
\text { - Not EHP number control } \\
\text { - EHP number control } \\
\text { - } \text { GHP number control } \\
\text { - Hybrid VRF System }\end{array}$ & $\begin{array}{l}\text { Setting } \\
\text { Air conditioning capacity } \\
\text { - } 100\left(\mathrm{~W} / \mathrm{m}^{2}\right) \\
\text { - } 150\left(\mathrm{~W} / \mathrm{m}^{2}\right) \\
\text { - } 200\left(\mathrm{~W} / \mathrm{m}^{2}\right)\end{array}$ \\
\hline - Calculate BEI and prima & $\begin{array}{l}\text { ion } \\
\text { ergy consumption }\end{array}$ \\
\hline
\end{tabular}

Fig. 8. Simulation flow chart

\subsection{Air-conditioning Zone}

Figure 9 shows the buildings floor plan. The area with color is the air-conditioning zone, the first floor is the lobby, the office 1, and office; the typical floor is office 1 , office 2 , and the conference room.

\subsection{Equipment specification}

Table 5 shows the equipment specifications. The composition of EHP and GHP is as follows: EHP has one motor of 12 horsepower and one of 18 horsepower, so a total of two units with 30 horsepower. GHP has two engines of 15 horsepower for a total of 30 horsepower. The integrated Hybrid VRF System has one EHP motor 10 horsepower and two GHP engines of 10 horsepower each, for a system total of 30 horsepower. In this paper, we input the order of EHP, GHP, GHP in order of unit number control, and simulated it as integrated Hybrid VRF System.

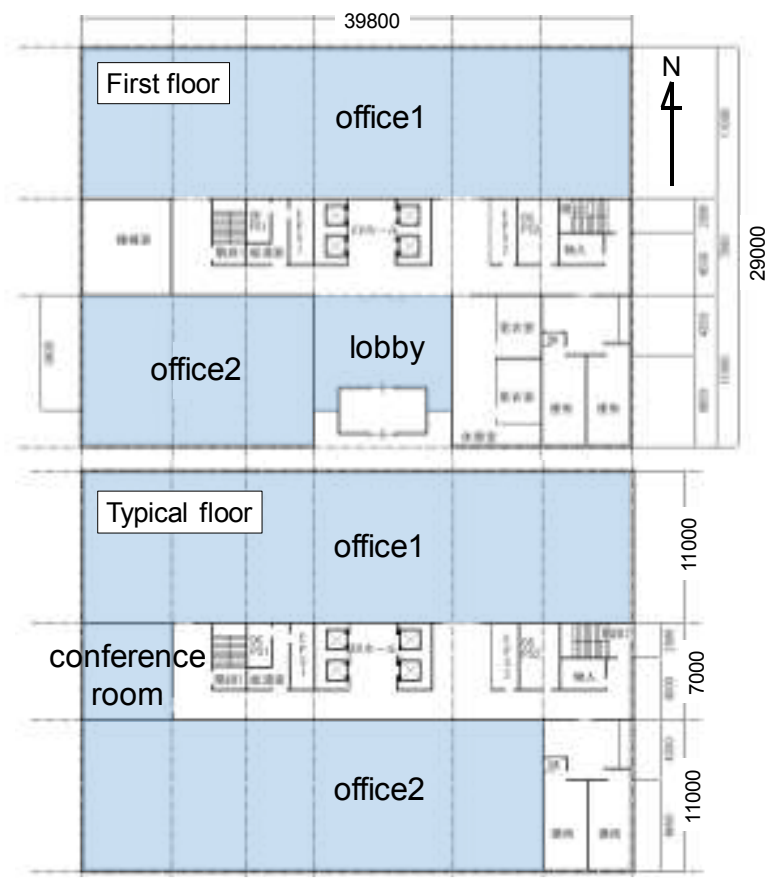

Fig. 9. Floor plan

Table 5. equipment specification

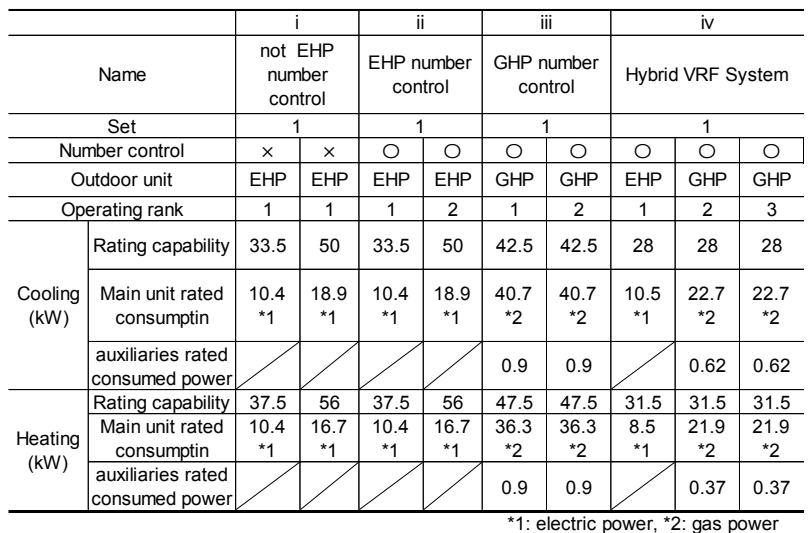




\subsection{System pattern in Heat source machine}

Figure 10 to 11 shows the system pattern of the outdoor unit by air-conditioning capacity. One system has 30 horsepower, so the air-conditioning capacity during cooling was even, and the air-conditioning zones were allocated for each verification pattern. The simulations to compare "not EHP number control," "EHP number control," "GHP number control," and the "Hybrid VRF System" were configured in the following outdoor unit patterns: set of 5 with $100\left(\mathrm{~W} / \mathrm{m}^{2}\right)$ capacity, set of 7 with $150\left(\mathrm{~W} / \mathrm{m}^{2}\right)$ capacity, and a set of 10 with a $200\left(\mathrm{~W} / \mathrm{m}^{2}\right)$ capacity.

\section{Ari conditioning capacity} : 200[W/m $]$

\section{Outdoor unit} : 10 set

\begin{tabular}{|l|l|}
\hline 10 & 9 \\
\hline 8 & 7 \\
\hline 6 & 5 \\
\hline 4 & 3 \\
\hline 2 & 1 \\
\hline
\end{tabular}

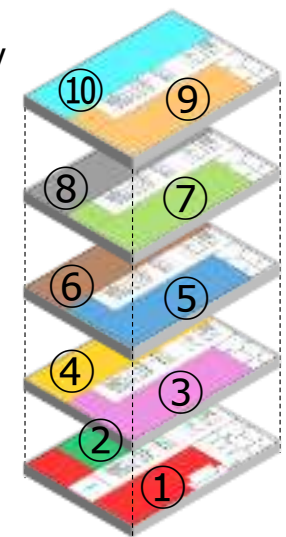

Fig. 10. Strain pattern at air-conditioning capacity of $200(\mathrm{~W} /$ $\left.\mathrm{m}^{2}\right)$

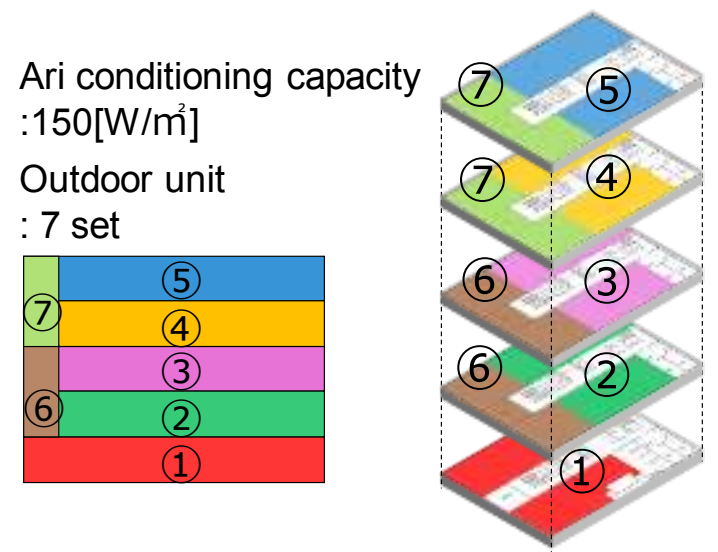

Fig. 11. Strain pattern at air-conditioning capacity of $150(\mathrm{~W} /$ $\left.\mathrm{m}^{2}\right)$

Ari conditioning capacity : 100[W/m²]

Outdoor unit $: 5$ set

\begin{tabular}{|c|}
\hline 5 \\
\hline 4 \\
\hline 3 \\
\hline 2 \\
\hline 1 \\
\hline
\end{tabular}

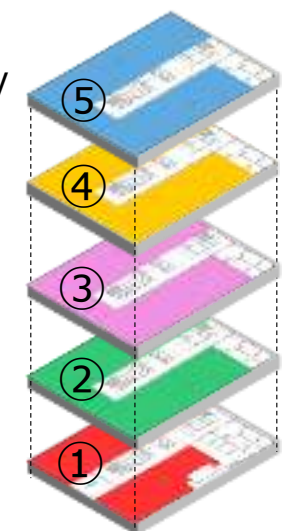

Fig. 12. Strain pattern at air-conditioning capacity of $100(\mathrm{~W} /$ $\left.\mathrm{m}^{2}\right)$

\section{Simulation results}

We used a Web Program. And the results of the relationship between BEI and primary energy consumption and primary energy consumption and load factor in the Hybrid VRF System with Not EHP unit number control, with EHP unit number control, with GHP number control is summarized.

\subsection{BEl and Primary energy consumption}

\subsubsection{The entire building}

Figure 13 shows the BEI and Primary energy consumption of the entire building. The reference value is a value automatically calculated by calculation method based on 3.2.1. ZEB Ready is achieved with BEI less than 0.5. ZEB Ready was achieved in all patterns except for the not control EHP number control of the airconditioning capacity of $200\left(\mathrm{~W} / \mathrm{m}^{2}\right)$.

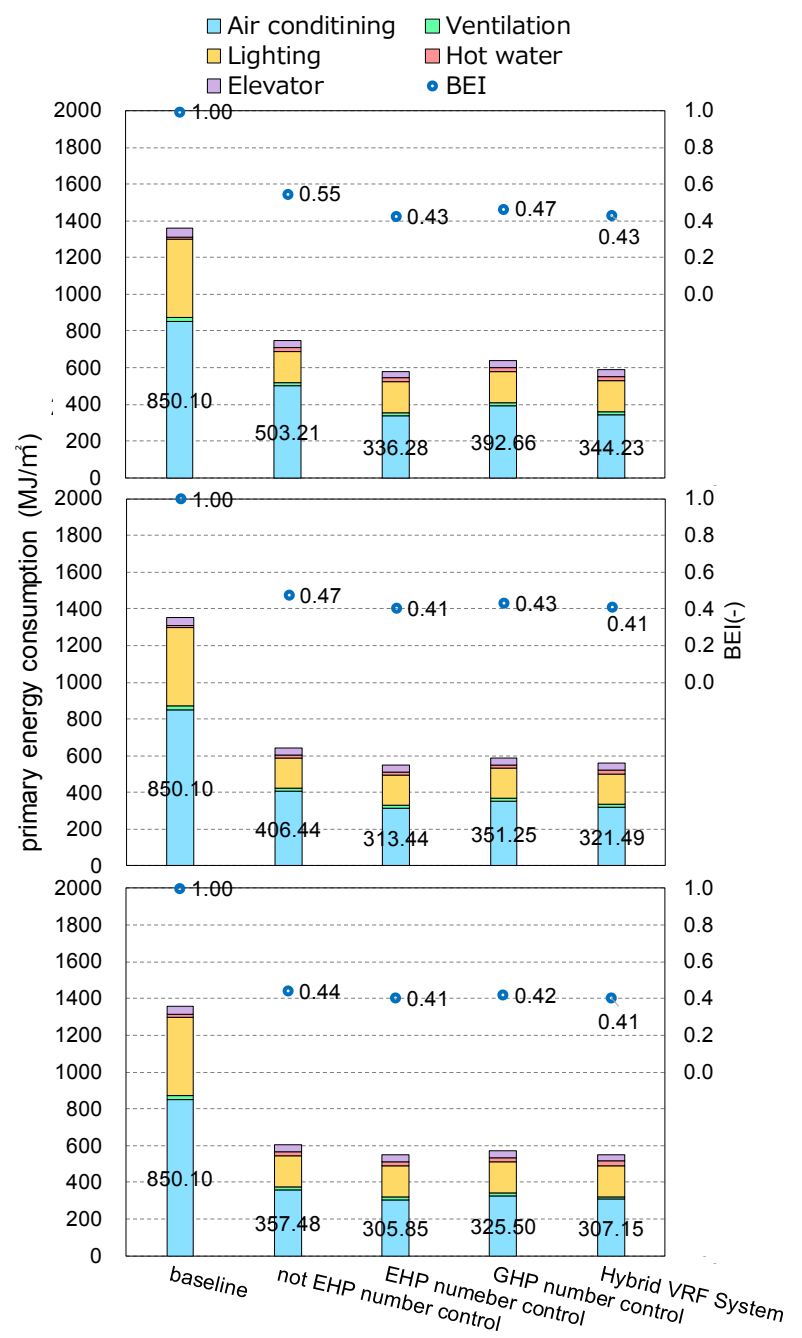

Fig. 13. BEI and Primary energy consumption (The entire building) 


\subsubsection{Air-conditioning}

Figure 14 shows the BEI and Primary energy consumption of the air-conditioning system. The primary energy consumption tends to decrease as the air conditioning capacity is narrowed down, and the operation ratio of GHP tends to increase in the integrated Hybrid VRF System.

\section{2 relationship between primary energy consumption and load factor}

Figure 15 shows the relationship between primary energy consumption and load factor of cooling and heating in each verification pattern. For air-conditioning, air-conditioning capacity $100\left(\mathrm{~W} / \mathrm{m}^{2}\right)$ is operating at the highest load factor. Primary energy consumption with EHP number control is small in any air -conditioning capacity in the load factor range of 0.1 to 0.3 , and the primary energy consumption of the Hybrid VRF System is small in the range of the load factor of 0.7 to the load factor of 1.0 -. As the vehicle operates at high loads, the second and third drives are performed according to the operating ranking of the integrated Hybrid VRF System. It is thought that it is influenced by GHP of Hybrid VRF System with higher rated COP than EHP with EHP number control. Heating is operating at a lower load factor than air-conditioning and the primary energy consumption of the Hybrid VRF System is the smallest among each verification pattern at any air -conditioning capacity.

\section{Conclusions}

In this paper, the integrated Hybrid VRF System was evaluated in a virtual medium sized office by the evaluation method specified by Japanese law. For the evaluation method, the author conducted a comparative verification in the case of introducing the same capacity EHP and GHP using Web Program. The findings obtained are shown below.

1) In the Web Program, the difference in primary energy consumption is caused by the influence of rated $\mathrm{COP}$ and unit number control.

2) Based on the results of BEI in the entire building, ZEB Ready was achieved with many verification patterns. In addition, almost equal evaluation was obtained from the results of EHP number control and integrated Hybrid VRF System.

3) Based on the results of BEI in the entire airconditioning system, the primary energy consumption becomes smaller in the case of controlling the number of units than in the case of not controlling the number of units and controlling the number of units. From this result, the significance of the number control can be seen

4) In the integrated Hybrid VRF System with air conditioning capacity of $100\left(\mathrm{~W} / \mathrm{m}^{2}\right)$ where high load operation was observed by downsizing, the primary energy consumption at high load is the smallest.

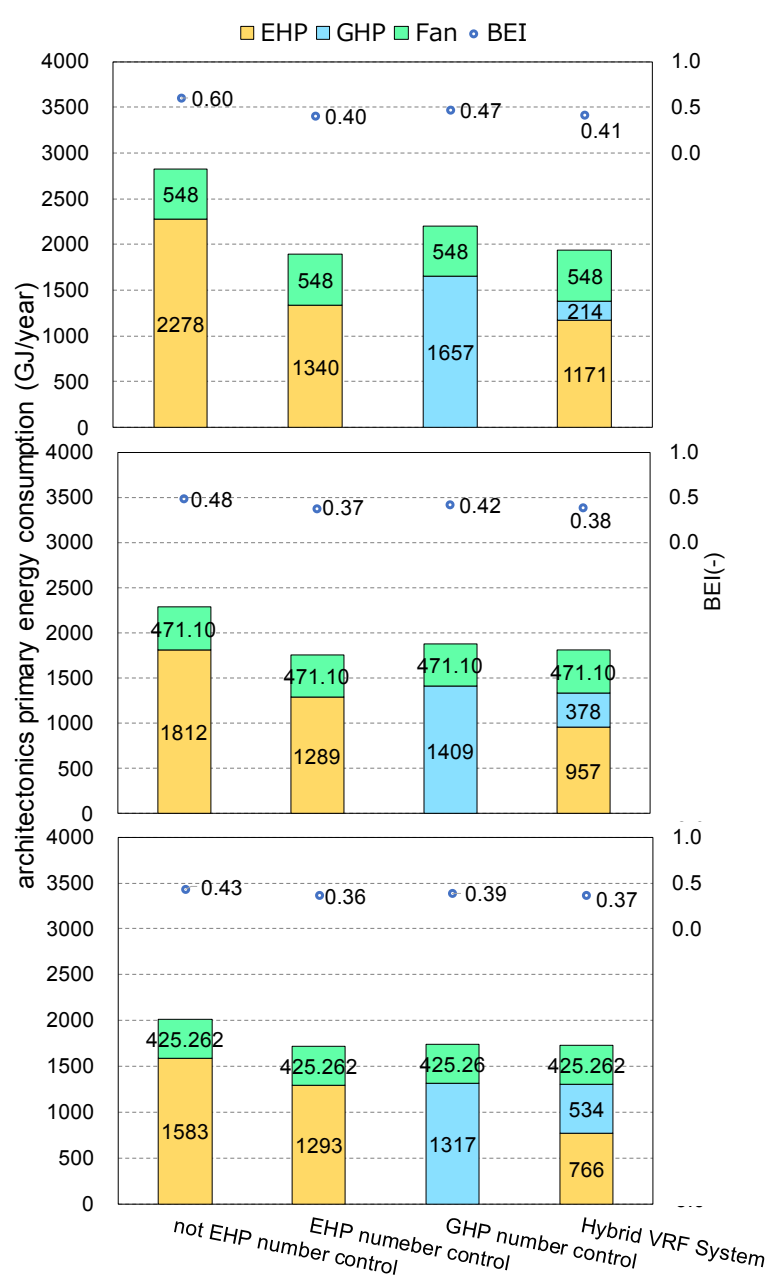

Fig. 14. BEI and Primary energy consumption (airconditioning system)

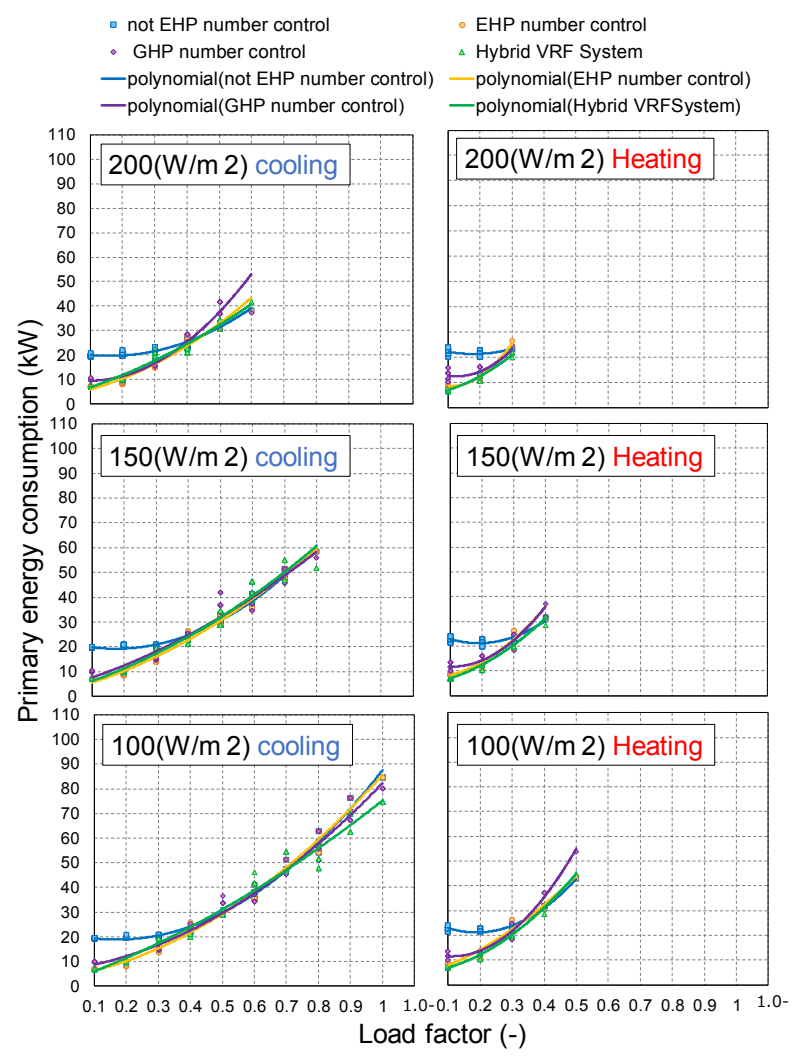

Fig. 15. Relationship between primary energy consumption 
1. Furuhashi Y., A Study on the Hybrid Airconditioning System having GHP and EHP in the Single Refrigerant Cycle Part 1 An Examination about the Most Optimal System Constitution by the Simulation [dissertation] Osaka, Japan: Air-Conditioning and Sanitary Engineers; 2015

2. Furuhashi Y., A Study on the Hybrid Airconditioning System having GHP and EHP in the Single Refrigerant Cycle Part 2 Verification of the Effectiveness of the Optimal Control in an Actual Building [dissertation]. Kagoshima, Japan: Air-Conditioning and Sanitary Engineers; 2016

3. Hashimoto A, Operating Status Evaluation of Integrated Hybrid VRF system [dissertation] Proceedings of ECOS 2018 - The 31ST international conference, Portugal

4. ZEB Roadmap Follw-up Committee, ZEB Design Guidelines ZEB Ready Medium Office Version $\sim$ Ver.0. Japan

5. WEBPRO enrgy consumption performance calculation program, non-residential version. Available at $:<$ https://building.app.lowenergy.jp/> [accessed 10.18.2018]

6. National Research and Development Corporation Building Research. 2. Methodof caculating energy consumption performance Available at $:<$ https://www.kenken.go.jp/becc/documents/buil ding/Definitions/Webpro_Specification_01AC 180810.pdf> 